\title{
The Bactrocera dorsalis species complex: comparative cytogenetic analysis in support of Sterile Insect Technique applications
}

\author{
Antonios A Augustinos ${ }^{1,2,3}$, Elena Drosopoulou', Aggeliki Gariou-Papalexiou', Kostas Bourtzis², \\ Penelope Mavragani-Tsipidou ${ }^{4}$, Antigone Zacharopoulou ${ }^{1 *}$
}

\begin{abstract}
Background: The Bactrocera dorsalis species complex currently harbors approximately 90 different members. The species complex has undergone many revisions in the past decades, and there is still an ongoing debate about the species limits. The availability of a variety of tools and approaches, such as molecular-genomic and cytogenetic analyses, are expected to shed light on the rather complicated issues of species complexes and incipient speciation. The clarification of genetic relationships among the different members of this complex is a prerequisite for the rational application of sterile insect technique (SIT) approaches for population control.

Results: Colonies established in the Insect Pest Control Laboratory (IPCL) (Seibersdorf, Vienna), representing five of the main economic important members of the Bactrocera dorsalis complex were cytologically characterized. The taxa under study were B. dorsalis s.S., B. philippinensis, B. papayae, B. invadens and B. carambolae. Mitotic and polytene chromosome analyses did not reveal any chromosomal characteristics that could be used to distinguish between the investigated members of the $B$. dorsalis complex. Therefore, their polytene chromosomes can be regarded as homosequential with the reference maps of $B$. dorsalis s.s.. In situ hybridization of six genes further supported the proposed homosequentiallity of the chromosomes of these specific members of the complex.

Conclusions: The present analysis supports that the polytene chromosomes of the five taxa under study are homosequential. Therefore, the use of the available polytene chromosome maps for B. dorsalis s.s. as reference maps for all these five biological entities is proposed. Present data provide important insight in the genetic relationships among the different members of the B. dorsalis complex, and, along with other studies in the field, can facilitate SIT applications targeting this complex. Moreover, the availability of 'universal' reference polytene chromosome maps for members of the complex, along with the documented application of in situ hybridization, can facilitate ongoing and future genome projects in this complex.
\end{abstract}

\section{Background}

The Bactrocera dorsalis complex species is a group of true fruit flies belonging to Tephritidae, with great economic importance. Following the most recent taxonomic revisions, this complex is currently harboring approximately 90 morphological similar taxa [1,2]. Among them, eight are considered as economic important pests [2], including among others B. dorsalis s.s., B. philippinensis, B. papayae and $B$. carambolae. In 2003, an addition to the complex

\footnotetext{
* Correspondence: zacharop@upatras.gr

'Department of Biology, University of Patras, Greece

Full list of author information is available at the end of the article
}

was made: $B$. invadens was detected in Kenya, and initially was considered a morphological variant of $B$. dorsalis s.s. [3]. However, in the following years it was recognized as a different species within the $B$. dorsalis complex [4]. Ever since that revision in 2005 , there were doubts regarding whether all these members really represent well-differentiated species, mainly due to the lack of robust diagnostic characters [5].

In recent years, accumulating data cast doubt on the 'actual' number of different species in the complex. Research performed by different laboratories points to a possible overestimation in the number of discrete taxa in the complex and the need of another taxonomic revision

\section{C) Biomed Central}


to incorporate the synonymic status of different species. This research includes morphological/morphometric studies [6-10], behavioral/sexual compatibility analysis $[11,12]$, as well as chemoecological $[13,13-15]$ and molecular genetic approaches [7-9,13,16-20]. Recently, Drew and Romig [1] have synonymized B. papayae with $B$. philippinensis; however there is also an ongoing debate about the species status of other important pests of the complex.

The delimitation of species within the $B$. dorsalis complex is not just a scientific question regarding evolution and speciation. It is also important for the agricultural economies of countries that heavily rely on fruit exports. The first aspect refers to quarantine measures. The current taxonomy leads to the implementation of certain quarantine policies; therefore it is critical to be as accurate as possible, when assessing species limits of economic important pest populations. As a characteristic example, the description of $B$. invadens as a separate species within the $B$. dorsalis complex prompted additional fruit export restrictions in many African countries, leading to increased economic losses $[7,12]$. The second aspect involves the effectiveness of SIT applications. SIT is probably the most environmental friendly pest control method since it is species specific and does not result in chemical or biological pollution. The main principle of SIT is the release of sterile flies in the field. Mating of sterile laboratory flies with the targeted population leads to infertile crosses and subsequent population suppression. Successful SIT is facilitated by a) the clarification of genetic relationships among targeted populations and laboratory strains and b) the availability of well characterized, stable and competitive genetic sexing strains (GSSs) that allow the release of only males into the field. The importance of stable and competing GSSs in SIT is well documented in the Tephritidae SIT model organism, Ceratitis capitata [21-23]. In principle, male only releases are more effective since they can lead to a) increased efficiency of sterile males in the field and b) better fruit quality, avoiding damage from released females. Today, there are only few GSSs for the B. dorsalis complex, initially developed for the control of $B$. dorsalis s.s. [24-26]. The creation of such strains through classical genetic approaches is species-specific and not an easy task. Thus, exploring the possibility of universal use of the same GSS for some of the economic important members of the complex could facilitate their control. The promising results of $[27,28]$, showing the possibility of controlling B. carambolae with $B$. dorsalis s.s. sterile flies point in such a direction.

Species limits can sometimes be obscure, and speciation can be driven by a variety of forces. Among them, chromosomal rearrangements (mainly inversions), are considered as key factors in Diptera speciation, especially in sympatric populations [29]. Early cytogenetic studies in Drosophila, based on mitotic and polytene chromosomes, were the first to detect interspecific inversions' differences [30,31]. Sturtevant and Dobzhansky [32] and Dobzhansky [33] first showed that chromosome inversions can be used to study the evolutionary history of a species group. Within this frame, inversions were proposed to have an important role in genetic variation and speciation leading thus to their extensive use as interspecific phylogenetic markers. The recent accumulation of comparative genomic data from Drosophila species [34-38] and mosquitoes [39-41] supports the importance of inversions in the suppression of gene flow in hybridizing taxa. Many models had been proposed regarding how inversions can enforce or support speciation, focusing mainly in the fitness of heterokaryotes (for a review see [42]). More recent theories, supported by genomic data, point to the suppression of recombination within and near inversions as a mechanism leading to reduced gene flow and maintenance of genetic divergence $[38,42,43]$. A possible role of an inversion can be the 'protection' of a combination of locally co-adapted alleles from introgression [44], that can lead to further accumulation of differences and facilitate speciation.

Taking into account the above, it is evident that cytogenetic analyses can help in resolving species boundaries within species complexes. This has been well documented in different Drosophila species [45], such as the endemic Hawaiian picture-winged group [46] and the American repleta species group [47]. In respect to this, the availability of polytene chromosomes in different Tephritidae genera, like Ceratitis [48], Bactrocera [49-53], Dacus [54], Rhagoletis [55-57,57] and Anastrepha [58] is valuable when seeking characteristic and diagnostic differences in closely related species.

Studies in B. dorsalis complex have also demonstrated the importance of adequate and well characterized samples: when exploring species limits and characters that may overlap, it is important to develop well organized and comprehensive sampling schemes $[8,16]$. Since species limits can be fuzzy and different classes of markers can provide different levels of resolution, the use of all available tools for species identification is highly desirable.

In the present study, we tried to identify chromosomal differences between five of the main agricultural pests of the complex, namely B. dorsalis s.s., B. philippinensis, $B$. papayae, B. invadens and B. carambolae, through the analysis of their mitotic complements and the comparison of their polytene chromosomes with the published reference maps for $B$. dorsalis s.s. [50]. As working material, samples representing well characterized colonies of these species, held at the Insect Pest Control Laboratory (IPCL, Seibersdorf, Vienna), were used. 
These colonies have been used in a variety of FAO/ IAEA projects $[8,11-13,16]$, and their status has been verified repeatedly. Polytene chromosomes derived from two $\mathrm{F}_{1}$ bidirectional hybrids $(B$. dorsalis s.s. $\times$ B. invadens and B. dorsalis s.s. $\times$ B. carambolae) were also analyzed, aiming at the detection of fixed chromosomal rearrangements among the parental colonies. We focused on these hybrids since: a) $B$. invadens is the only member of the complex originating from Africa, and its current recognition as a distinct species within the complex has severe quarantine consequences and $b$ ) $B$. carambolae is considered to be more clearly differentiated from the other four members of the complex $[10,11,16]$. Finally, in situ hybridization was performed using unique genes, attempting to: a) provide diagnostic landmarks for the polytene chromosome arms, b) reveal small chromosome rearrangements undetectable by microscopic observation and c) test the utility of $B$. dorsalis complex polytene chromosomes and polytene maps for future mapping experiments.

\section{Methods}

\section{B. dorsalis complex strains}

Colonies representing the five economic important members of the complex currently established at the IPCL were used. Specifically, two colonies of $B$. dorsalis s.s. (Saraburi - Thailand and Nakhon Si Thammarat Thailand), one of B. philippinensis (Philippines), one of $B$. papayae (Serdang-Malaysia), one of B. invadens (Kenya) and one of B. carambolae (Paramaribo, Suriname) were analyzed. In addition, the two following $\mathrm{F}_{1}$ bidirectional hybrids were analyzed: a) B. dorsalis s.s. (Saraburi strain) $\times$ B. carambolae and b) B. dorsalis s.s. (Saraburi strain) $\times$ B. invadens.

\section{Mitotic chromosome preparations}

Chromosome preparations were made, as described in [48]. Brain tissue from third instar larvae was dissected in $0.7 \% \mathrm{NaCl}$, transferred to $1 \%$ sodium citrate on a well slide for at least $15 \mathrm{~min}$ and fixed in fresh fixation solution (methanol/acetic acid 3:1) for 3min (fixation solution was changed twice in this step). Fixation solution was removed and a drop of acetic acid (60 \%) was added. Tissue was dispersed using a micropipette and the cell suspension was dried by laying it on a clean slide placed on a hotplate (40$45{ }^{\circ} \mathrm{C}$ ). Chromosomes were stained with Giemsa (5\% Giemsa in $10 \mathrm{mM}$ phosphate buffer, $\mathrm{pH}$ 6.8). Chromosome slides were analyzed at $100 \times$ magnification, using a phase contrast microscope (Leica DMR), and photographs were taken using a CCD camera (ProgRes CFcool; Jenoptik Jena Optical Systems, Jena, Germany). At least 15 good quality preparations (each one representing one larva) per sample and at least 10 well spread nuclei per preparation were analysed.

\section{Polytene chromosome preparations}

Polytene chromosome preparations were made from $3^{\text {rd }}$ instar larvae, as described in [48]. Larvae were dissected in acetic acid ( $45 \%)$, and salivary glands were transferred to $\mathrm{HCl}(3 \mathrm{~N})$ for $1 \mathrm{~min}$, fixed in 3:2:1 fixation solution ( 3 parts acetic acid: 2 parts water: 1 part lactic acid) for $\sim 5 \mathrm{~min}$ (until transparent) and stained in lactoacetic orcein for 5-7 min. Glands were washed with 3:2:1 solution to remove excess stain and squashed. Chromosome slides were analyzed at $100 \times$ magnification using a phase contrast microscope (Leica DMR) and photographs were taken using the ProgRes CFcool CCD camera. At least 25 good quality preparations (each one representing one larva) per sample and at least 10 well spread nuclei per preparation were analysed.

\section{In situ hybridization}

Polytene chromosome preparations for in situ hybridization were made from salivary glands of 1-4 day-old pupae, as described in [59]. Six heterologous gene sequences originating from other tephritid species were used as probes (Table 1). Labeling and detection was performed using the DIG DNA Labeling and Detection kit (ROCHE Diagnostics, Mannheim, Germany), according to [60]. Hybridization was performed at $60{ }^{\circ} \mathrm{C}$. Two to three preparations per strain were hybridized with each probe, and at least ten well spread nuclei per preparation were analyzed.

\section{Results}

\section{Mitotic karyotype analysis}

All the members of the complex analyzed here $(B$. dorsalis s.s., B. philippinensis, B. papayae, B. invadens and B. carambolae) show five pairs of autosomes and one pair of heteromorphic sex chromosomes (XX/XY). The autosomes have been numbered II to VI according to descending size order [50]. The two longest (II and III) and the two shortest (V and VI) autosomes can be described as submetacentric, although with different arm ratios, and one autosome (IV) can be described as metacentric. The sex chromosomes are the smallest of the set, with the $\times$ being elongated, metacentric, with one of the arms being darker stained than the other and the $\mathrm{Y}$ being dot-like (Figure 1). The observed karyotype is referred as form A [61]. No differences in the karyotypes were observed.

\section{Polytene chromosome analysis}

No evidence of polytenization of the sex chromosomes was observed. This in accordance with the polytene complement published for B. dorsalis s.s. [50].

A comparison of the polytene elements of all analyzed strains with the reference map of $B$. dorsalis s.s. [50] revealed perfect correspondence of the banding patterns. 
Table 1 The hybridization probes used in the present study and their localization sites on the polytene chromosomes of the five taxa studied from the $B$. dorsalis species complex.

\begin{tabular}{|c|c|c|c|c|c|}
\hline Gene name & Description & Species of origin & DNA type & Reference & Localization site \\
\hline hsp70 & the heat-shock 70 gene & Ceratitis capitata & genomic & [74] & $26-3 L$ \\
\hline gld & the glutamate dehydrogenase gene & Ceratitis capitata & genomic & unpublished & $6-2 \mathrm{~L}$ \\
\hline scarlet & the orthologue of the scarlet gene of D. melanogaster & Bactrocera tryoni & genomic & [75] & $82-6 \mathrm{~L}$ \\
\hline ovo & orthologue of the ovo gene of D. melanogaster & Bactrocera oleae & CDNA & unpublished & $63-5 L$ \\
\hline$s x l$ & orthologue of the sex lethal gene of D. melanogaster & Bactrocera oleae & CDNA & [76] & $78-5 R$ \\
\hline tra & orthologue of the transformer gene of $D$. melanogaster & Bactrocera oleae & cDNA & [77] & $86-6 \mathrm{~L}$ \\
\hline
\end{tabular}

The localization site was determined according to the $B$. dorsalis s.s. polytene maps [50]

No fixed chromosome rearrangements were detected. Consequently, all the strains can be regarded as homosequential and the available polytene chromosome maps of B. dorsalis s.s. can be used for all of them. Furthermore, the heterochromatic mass of the centromeric regions was identical in quality and quantity in all analyzed members of the complex, providing a useful landmark for the identification of each polytene chromosome. The characteristic polymorphic asynapsis on the right arm of chromosome 5 (sections 73-74), previously found in B. dorsalis s.s. [50], was also observed at varying frequencies (10-50 \%) in all samples (Figure 2a-c). A few additional minor polymorphic asynapses were distributed over the polytene arms (Figure 2d-e).

Polymorphic inversions were found on chromosome arm $2 \mathrm{R}$ of the two B. dorsalis s.s. samples (Figure 3). This is in accordance with the data of [50]. No other members of the complex showed any polymorphic inversions.

\section{Polytene chromosome analysis of $F_{1}$ hybrids}

In order to verify the identical banding pattern of the analyzed species, cytological analysis of $\mathrm{F}_{1} B$. dorsalis s.s. $\times B$. invadens (bidirectional) and $\mathrm{F}_{1}$ B. dorsalis s.s. $\times$ B. carambolae hybrids (bidirectional) was performed. The analysis
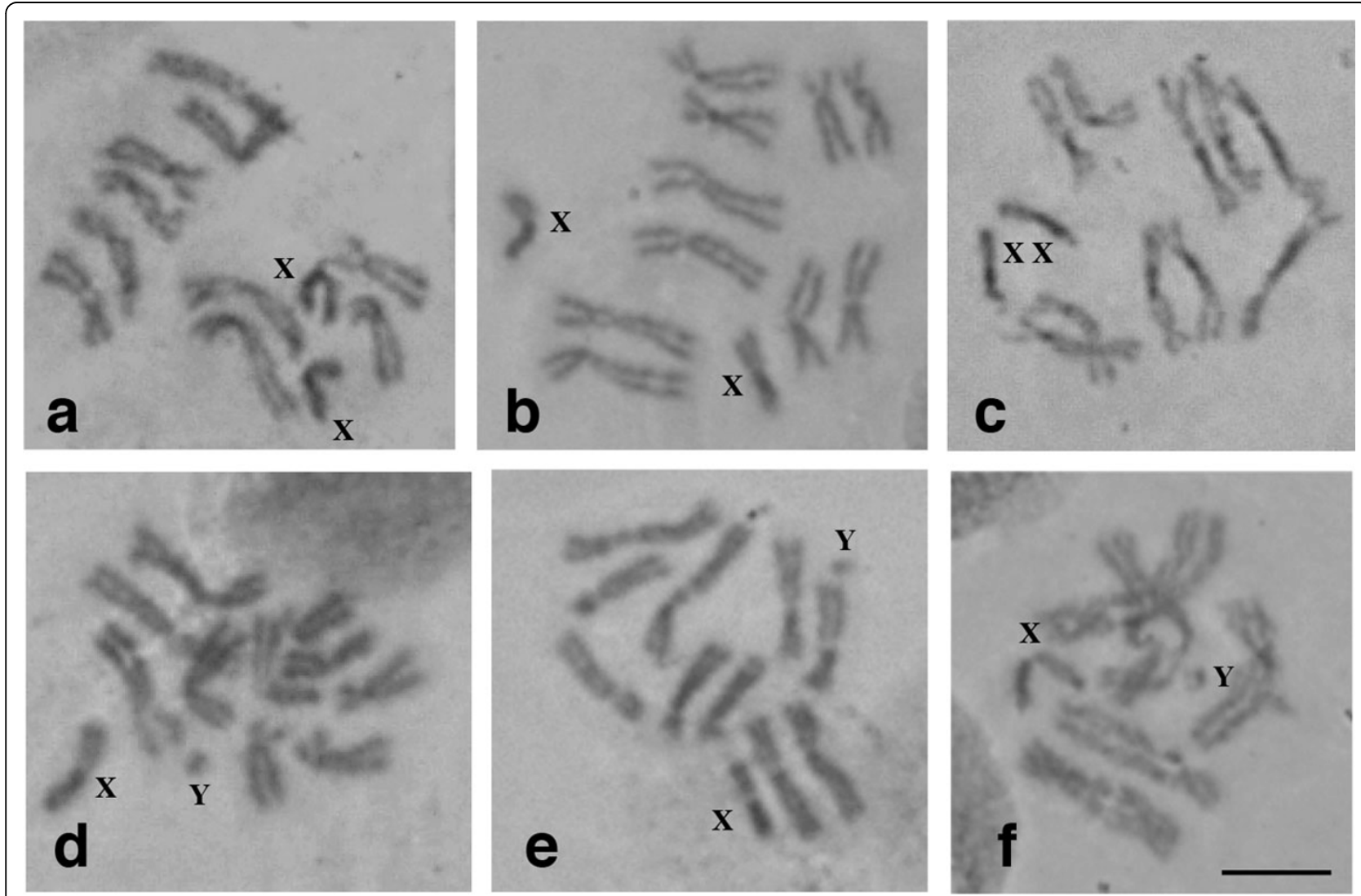

Figure 1 Mitotic karyotypes of members of the $\boldsymbol{B}$. dorsalis species complex. a and d) B. dorsalis s.s. (Saraburi), b) B. papayae, c) B. invadens, e) B. carambolae, f) B. philippinensis. a-c) females, $\mathbf{d}-\mathbf{f})$ males. Scale bar represents $5 \mu \mathrm{m}$. 

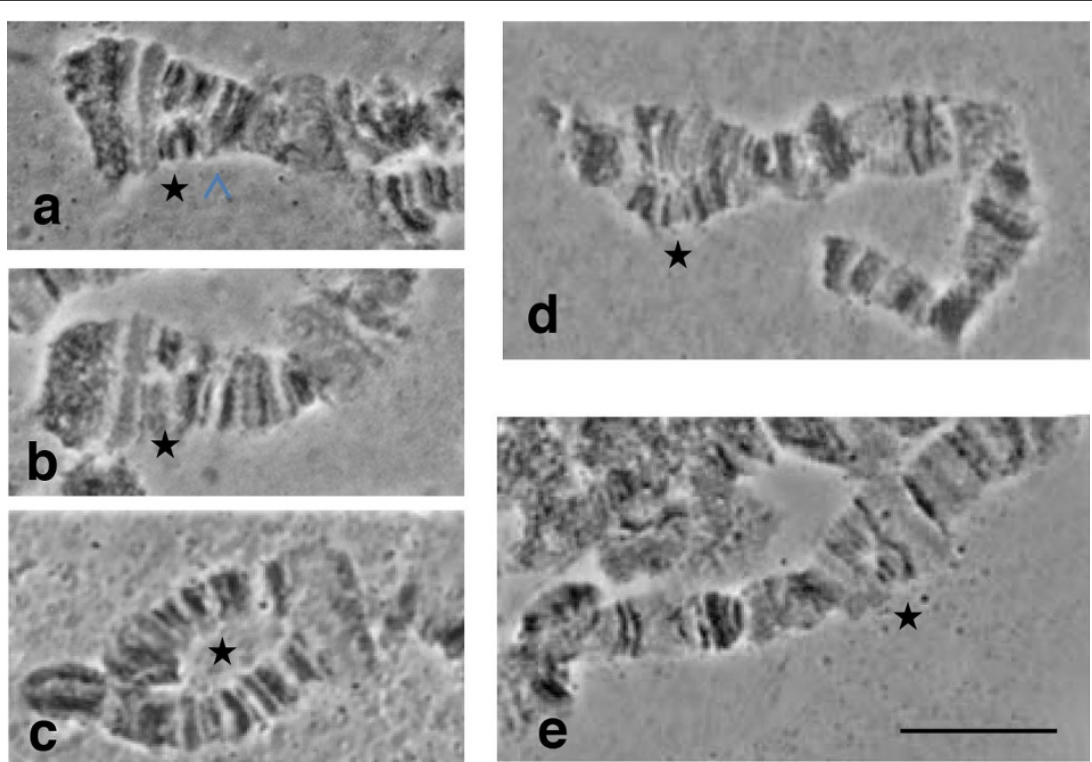

Figure 2 Polymorphic asynapses in different polytene chromosome regions. Variations in the appearance of the asynaptic region $73-74$ of chromosome arm 5R: a) in B. carambolae and (b, c) in the B. dorsalis s.s. $\times$ B. carambolae hybrid. Minor asynapses in the B. dorsalis s.S. $\times$ B. carambolae hybrid within: $\mathbf{d}$ ) regions 43 of chromosome arm $4 \mathrm{~L}$ and e) regions 78-79 of chromosome arm 5R. Asterisks indicate the asynaptic regions. Scale bar represents $10 \mu \mathrm{m}$.

of chromosome preparations of the hybrids did not reveal signs of fixed chromosome differences between the parental strains, evident from the perfect synapses among the parental homologous chromosomes (Figure 4). The comparison with the reference polytene chromosome maps of $B$. dorsalis s.s. verified that hybrids and their parental strains are homosequential with B. dorsalis s.s. (Figure 5). In both hybrids, similar to the parental strains, the asynapsis at region 73-74, together with some other minor polymorphic asynapses were observed (Figure 2b-e). The number of minor asynaptic sites was higher in the $B$. dorsalis s.s. $\times$ B. carambolae $\mathrm{F}_{1}$ hybrids than the B. dorsalis s.s. $\times$ B. invadens $\mathrm{F}_{1}$ hybrids.

\section{In situ localization of genes}

In situ localization of six unique genes, namely $g l d$, hsp70, ovo, sxl, scarlet and tra (Table 1) was performed on the polytene chromosomes of the five taxa, as well as on the two hybrids. Each probe yielded a unique signal at the same chromosomal position in all entities. More specifically, gld localized in region 6 of $2 \mathrm{~L}, h s p 70$ in region 26 of $3 \mathrm{~L}$, ovo in region 63 of $5 \mathrm{~L}, S x l$ in region 78 of $5 \mathrm{R}$ and scarlet and tra in regions 82 and 83 of arm 6L (Table 1, Figure 6).

\section{Discussion}

The main findings of the present study can be summarized as follows: a) mitotic karyotypes of the five members of the complex presented form A, the typical one for B. dorsalis s.s. which, according to [61] represents the most ancestral form of the complex, b) polytene chromosome analysis of both parental strains and selected $F_{1}$ hybrids did not reveal any fixed differences among the five members of the complex, and c) in situ hybridization of selected genes confirmed that there are no differences among the five members of the complex based at least on the limited number of probes tested. The in situ results also provided characteristic landmarks for the recognition of the polytene arms and demonstrated the utility of polytene chromosomes and reference maps of the complex for in situ mapping projects.

\section{Implications for SIT applications}

The $B$. dorsalis species complex includes at least eight economic important pests [2] that infest a variety of hosts worldwide and are putative targets for SIT. The development of GSSs, a prerequisite for efficient and cost-effective SIT programs, has been already achieved for B. dorsalis s.s. [24-26]. However, the availability of such strains does not mean that they are $a$ priori suitable for mass rearing and release purposes. These strains have to exhibit a number of traits, such as genetic stability and good productivity in the laboratory or in mass rearing conditions over a number of generations as well as male mating competitiveness in the field. In respect to this, cytogenetic knowledge of the chromosomal events generating GSSs, along with standard Quality Control (QC) measures are of major importance for the assessment of the abovementioned traits [62]. 

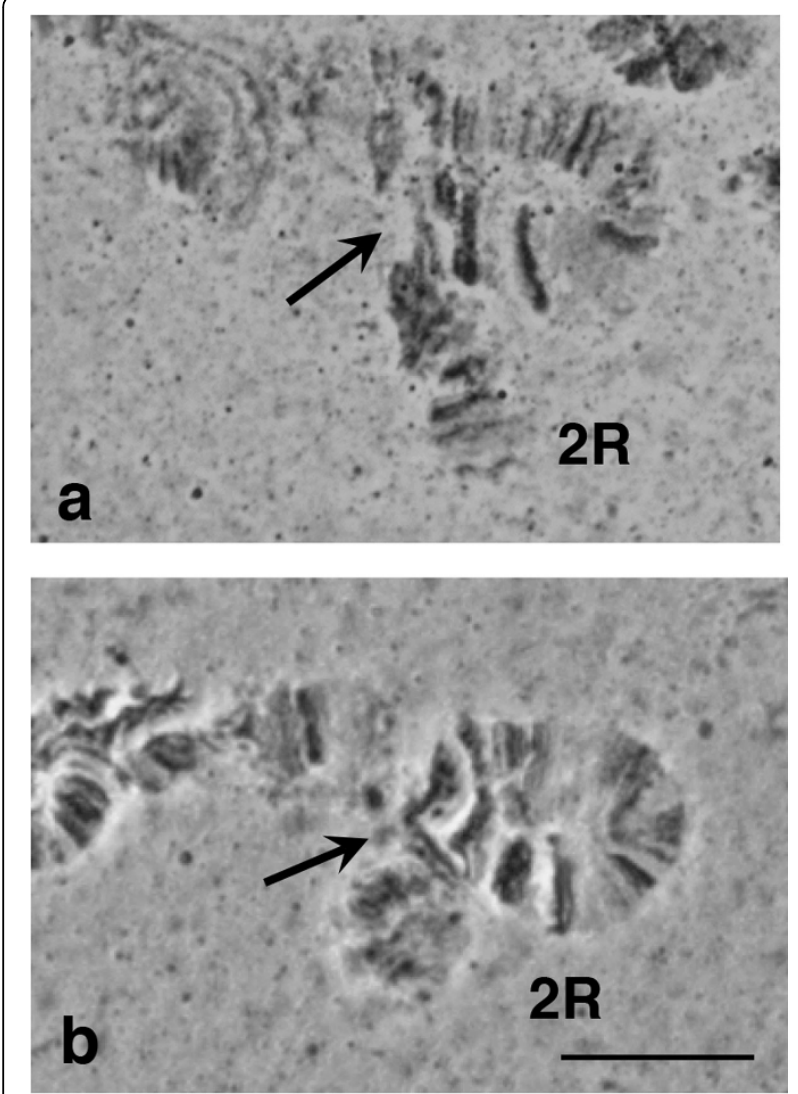

Figure 3 The polymorphic inversion on the $2 R$ polytene chromosome arm. a) A polymorphic inversion found in the distal part of chromosome arm $2 R$ in the Saraburi colony of $B$. dorsalis s.s., b) the same inversion in the $B$. dorsalis s.s. $\times$ B. invadens hybrid. Arrows indicate the breakpoints. Scale bar represents $10 \mu \mathrm{m}$.

The resolution of biological relationships among the different entities of species complexes is of high importance, since SIT application without this knowledge could jeopardize the effectiveness of such programs, especially in areas where different members of the complex overlap. This information is very useful in respect to the selection of appropriate laboratory strains for release purposes. The findings of the present study along with other studies that support a single species scenario $[7-9,11,13,16,17]$ at least for the four of the five economic important members of the complex studied (B. dorsalis s.s., B. papayae, B. philippinensis and $B$. invadens), favour the 'universal' application of the B. dorsalis s.s. GSSs against all the above members of the complex. This is very important, considering the effort required in generating GSSs through classical genetic methods. In respect to this, the recent study of [27] presented in the same special issue points to the possibility of using the B. dorsalis s.s. GSS against B. carambolae, after several generations of crosses aiming to integrate this strain to B. carambolae genomic background.

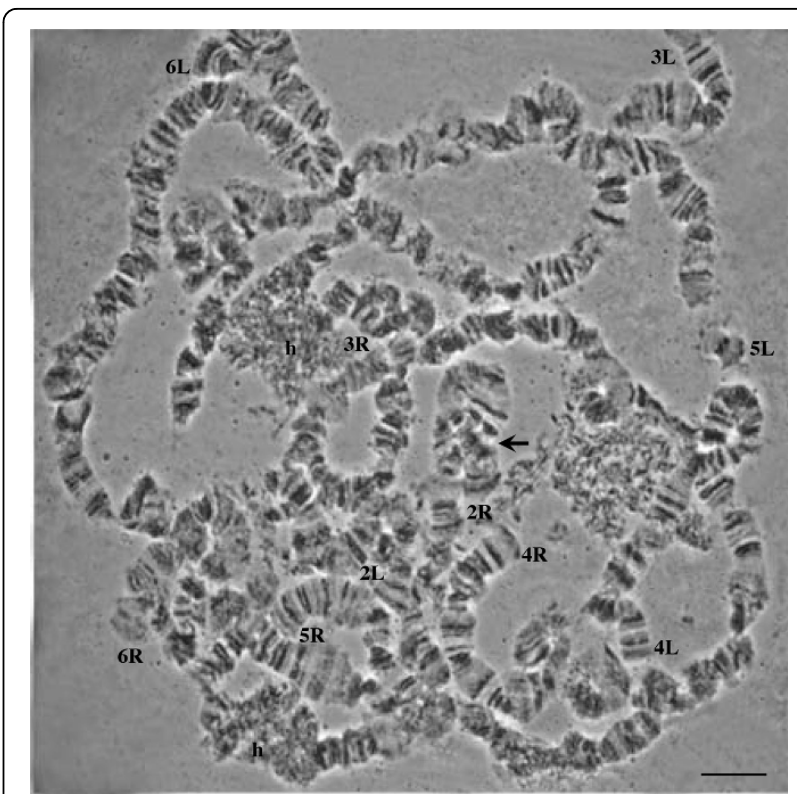

Figure 4 Polytene nucleus of the $F_{1}$ hybrid of $B$. dorsalis s.s. $\times$ B. carambolae. Note the perfect synapsis along the parental homologous chromosomes. Arrow indicates the polymorphic inversion found in the distal part of $2 \mathrm{R}$ chromosome arm in the Saraburi colony of $B$. dorsalis s.s. The chromosome tips are indicated; $h$ indicates pericentric heterochromatin. Scale bar represents $10 \mu \mathrm{m}$.

\section{Mitotic karyotypes - no evidence of differentiation}

Our analysis of mitotic chromosomes shows that all members of the complex studied here exhibit the same karyotype, described previously as form A [61]. This form is assumed to be the ancestral form in the complex and typical of B. dorsalis s.s. [50,61,63-65]. However, Baimai et al. [63] had previously described a different mitotic karyotype for B. carambolae. In that study, samples derived directly from infested fruits and characterized as B. carambolae based on morphological, geographic and host criteria, were reported to possess $\times$ chromosomes larger than the autosomes (form E karyotype). Our analysis does not confirm this report. A recent cytogenetic study on a B. carambolae colony derived from Malaysia also presented a typical form A karyotype for this species [66].

Given that a) geographic origin and plant host alone cannot be regarded as absolute taxonomic criteria $[9,10,19]$ and $b)$ it is difficult to establish robust morphological diagnostic characters for the different members of the complex $[7,9,13,16,17,19]$, it is apparent that one must be quite skeptical regarding accurate species identification based only on these parameters. To avoid such problems, in the present study we used only material from IPCL. This is colonized material and therefore available at any time for different types of analysis.

Previous studies on mitotic karyotypes of the $B$. dorsalis complex have shown that there is considerable variability in size and ratio of the $\mathrm{X}$ chromosome arms 


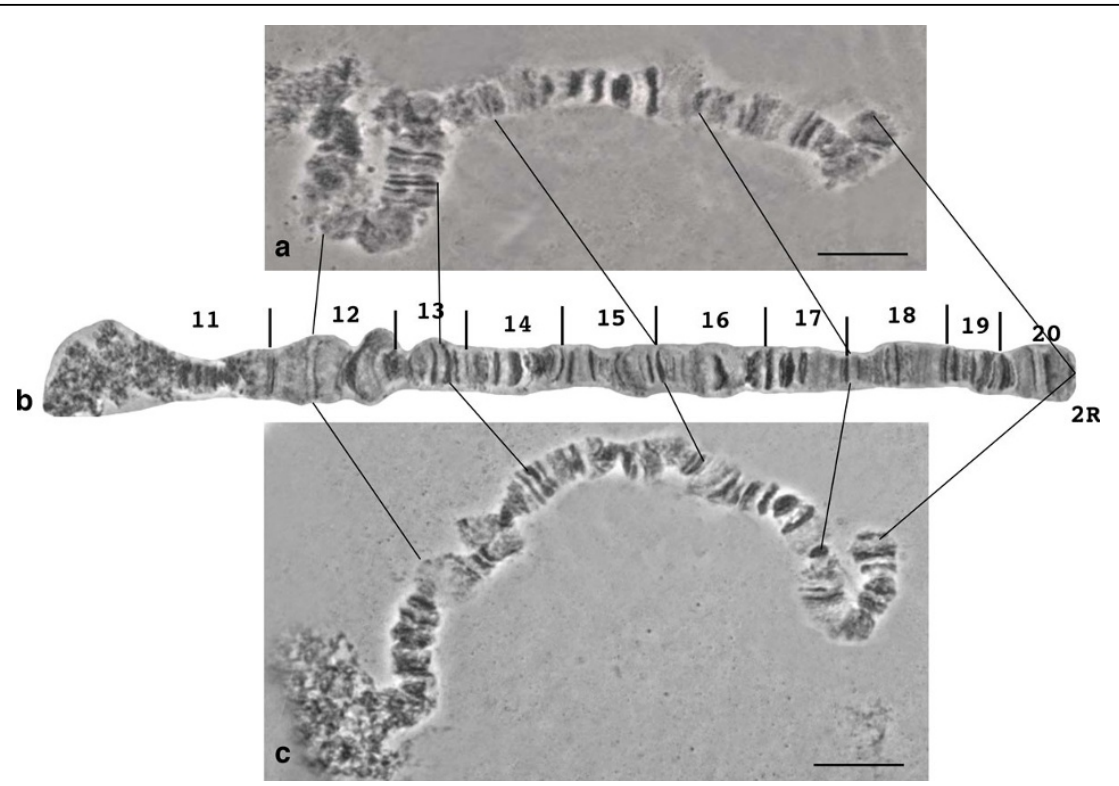

Figure 5 Comparison of the $2 \mathrm{R}$ chromosome arm between $B$. dorsalis s.s. and its hybrids with $B$. carambolae and $B$. invadens. a) chromosome arm $2 R$ of the $F_{1}$ hybrid of $B$. dorsalis s.s. $\times$ B. carambolae, $\mathbf{b}$ ) reference map of chromosome arm $2 R$ of B. dorsalis s.s. and $\mathbf{c}$ ) chromosome arm $2 \mathrm{R}$ of the $\mathrm{F}_{1}$ hybrid of $B$. dorsalis s.s. $\times$ B. invadens. Note the banding pattern similarity. Scale bar represents $10 \mu \mathrm{m}$.

[61,63-65]. X and Y size polymorphism has also been observed in other tephritid species complexes, including the A. fraterculus complex [67]. The highly heterochromatic nature of the sex chromosomes in all tephritids analyzed so far (evident also from the lack of polytenization due to their under-replication) $[48-51,53-58,68,69]$ points to a possible increased 'tolerance' in gain and loss of material in these chromosomes, which could explain its size plasticity.

\section{Polytene chromosome analysis - no evidence of} speciation mediated by chromosomal rearrangements The proposed chromosomal homosequentiality of the five members of the $B$. dorsalis species complex is based on the following observations: i) absence of fixed chromosomal rearrangements in comparison to the reference map of B. dorsalis s.s.; ii) absence of differences among the parental homologous chromosomes in the two hybrids studied; iii) identical heterochromatic mass of the centromeric regions of each chromosome element in all taxa; iv) common characteristic asynapsis of the chromosomal region 73-74 and v) in situ localization of each of six genes on the same chromosomal region in all taxa analyzed.

In tephritid flies, genomic data are still scarce, and polytene chromosome maps are restricted to a few species. However, comparative polytene chromosome analysis and

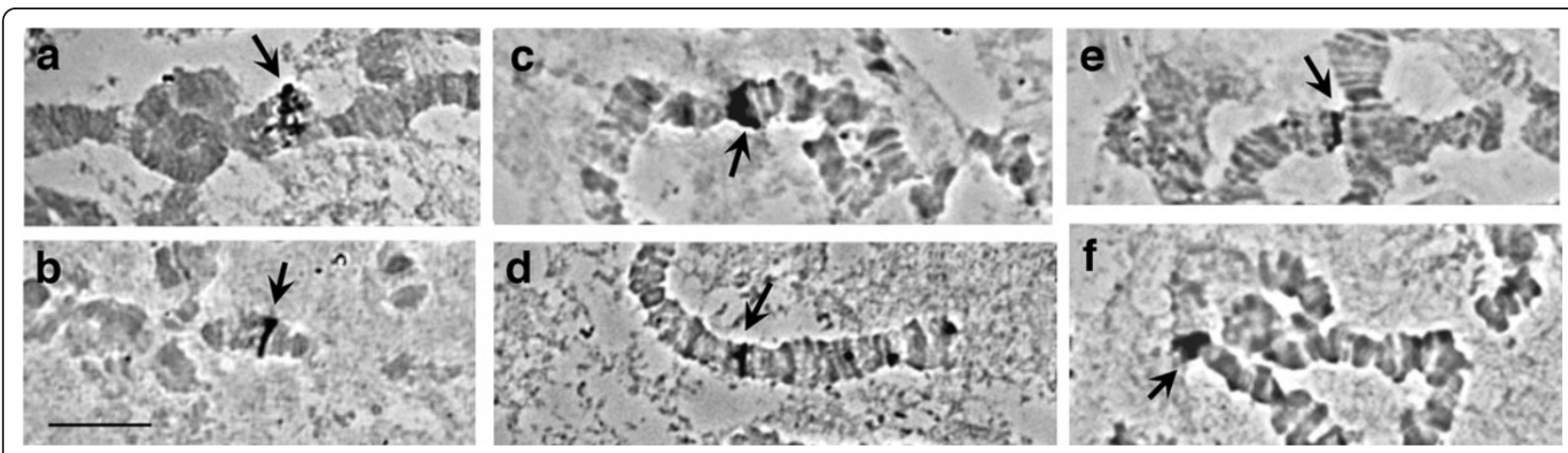

Figure 6 Hybridization sites of six different probes on salivary gland polytene chromosomes of the $B$. dorsalis complex species. a) gld in the B. dorsalis s.s. $\times$ B. invadens hybrid, b) hsp 70 in B. dorsalis s.s. $\times$ B. carambolae hybrid, c) ovo in B. dorsalis s.s. $\times$ B. carambolae hybrid, d) sXl in B. dorsalis s.s. $\times$ B. carambolae hybrid, e) scarlet in B. dorsalis s.s. and f) tra in B. dorsalis s.s. $\times$ B. carambolae hybrid. Arrows point to the hybridization signals. Note that signals in the hybrids show no differences between the two parental homologous chromosomes. Scale bar represents $10 \mu \mathrm{m}$. 


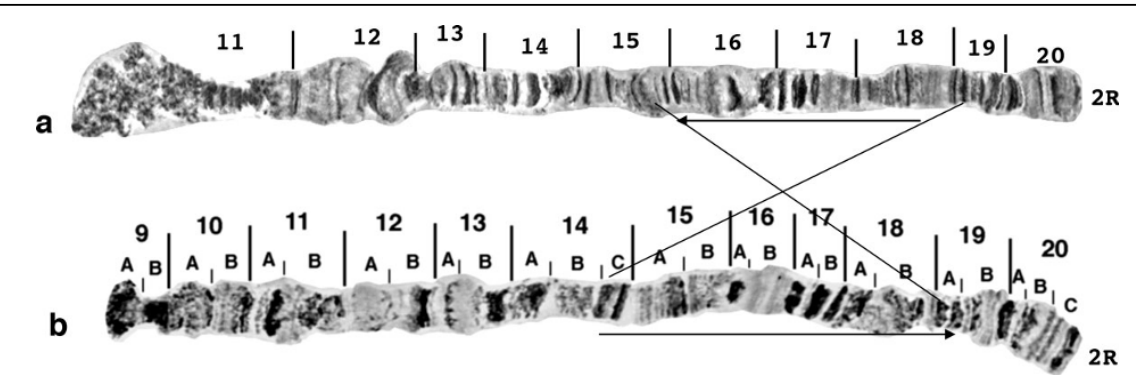

Figure 7 Comparison of chromosome arm 2R between B. dorsalis s.s. and B. tryoni. a) Chromosome arm $2 \mathrm{R}$ of $B$. dorsalis s.s. and b) chromosome arm $2 R$ of $B$. tryoni. Note the fixed inversion between the two species.

in situ mapping of unique genes show that chromosomal rearrangements characterize different species $[50,51$, $53,58,69]$, suggesting their possible involvement in speciation. A comparative analysis of polytene chromosome maps of B. dorsalis s.s. and B. tryoni, a species outside, but closely related to the $B$. dorsalis complex, clearly shows at least one fixed inversion in polytene arm $2 \mathrm{R}$ that differentiates the two species (Figure 7).

Even though fixed rearrangements were not found in the polytene chromosome of the species studied, polymorphic inversions were observed in the two B. dorsalis s.s. populations, as well as in the two hybrids (derived from the $B$. dorsalis s.s. genome). A similar observation has been reported for another Thailand B. dorsalis s.s. population [50]. Although not reported in other tephritids, polymorphic inversions are common in Diptera and their presence and frequencies usually differ between geographical populations within the species [31]. The cytogenetic analysis of more species of the $B$. dorsalis complex could provide important insight in the involvement of chromosomal rearrangements in speciation within this species group.

The minor polymorphic asynapses observed in all samples most probably represent differential gene expression of the two parental homologous chromosomes. However, the presence of small, undetectable (with microscopic observation) rearrangements, such as inversions, deletions or insertions of repetitive or heterochromatic material, cannot be excluded. Indeed, even small inversions can alter the control of regulatory elements and lead to differential gene expression (puffing activity) [43]. Thus, the higher number and frequency of small polymorphic asynapses observed in the $B$. dorsalis s.s. $\times$ B. carambolae hybrid, in respect to the $B$. dorsalis s.s. $\times$ B. invadens hybrid, may indicate that the $B$. carambolae genome has small differences compared to the other members of the complex. Current literature tends to support B. carambolae as a discrete entity within the complex, but closely related to the others $[10,13,16,19]$. The ability of $B$. carambolae to a) mate, although with reduced compatibility, b) produce viable and fertile progeny in the lab and c) produce hybrids carrying intermediate characteristics with other members of the complex $[11,28,70,71]$ points to the presence of mainly prezygotic isolation between $B$. carambolae and the other members of the complex. Therefore, this is a case most likely representing incipient rather than complete speciation, a phenomenon also observed in the A. fraterculus complex $[67,69]$.

\section{Conclusions}

The present study sheds important light in the delimitation of species boundaries within the $B$. dorsalis species complex. Our data are in accordance with other recent studies questioning the currently accepted number of discrete species within this complex, since no fixed chromosomal differences were found. This outcome is of major importance for SIT applications targeting the different members of the complex. Currently, there are efforts towards genome/transcriptome sequencing of the $B$. dorsalis complex [72,73] that are generating a great amount of sequences with, however, limited information regarding their overall organization. The comparative cytogenetic analysis presented here, accompanied with the in situ hybridization of genes on the polytene chromosomes, highlight the importance of cytogenetics in gaining more insight regarding organization of newly generated contig sequences and chromosomal localization of genes of specific interest.

\section{Competing interests \\ No competing interests exist.}

\section{Authors' contributions}

AAA, AGP, AZ conceived the experiments. AAA, ED, AGP, PMT, AZ performed the experiments. AAA, ED, AGP, KB, PMT, AZ performed the analysis. AAA, $E D, A G P, K B, P M T, A Z$ wrote the manuscript. All authors read and approved the manuscript.

\section{Acknowledgements}

The authors are grateful to the Joint FAO/IAEA Division of Nuclear Techniques in Food and Agriculture for the overall support on this study. We would also like to thank Franz G, Remboulakis Ch and Caceres C from Insect Pest Control Laboratory (Seibersdorf, Vienna) for their valuable support and the supply of the biological material used in this study. Finally, we would like to thank the two anonymous reviewers for their valuable comments that helped us prepare a really improved version of this manuscript 
This article has been published as part of BMC Genetics Volume 15 Supplement 2, 2014: Development and evaluation of improved strains of insect pests for SIT. The full contents of the supplement are available online at http://www.biomedcentral.com/bmcgenet/supplements/15/S2. Publication of this supplement was funded by the International Atomic Energy Agency. The peer review process for articles published in this supplement was overseen by the Supplement Editors in accordance with BioMed Central's peer review guidelines for supplements. The Supplement Editors declare that they have no competing interests.

\section{Authors' details}

${ }^{1}$ Department of Biology, University of Patras, Greece. ${ }^{2}$ Insect Pest Control Laboratory, Joint FAO/IAEA Programme of Nuclear Techniques in Food and Agriculture, Seibersdorf, Vienna, Austria. ${ }^{3}$ Department of Environmental and Natural Resources Management, University of Patras, Agrinio, Greece. ${ }^{4}$ Department of Genetics, Development and Molecular Biology, School of Biology, Faculty of Sciences, Aristotle University of Thessaloniki, Thessaloniki, Greece.

\section{Published: 1 December 2014}

\section{References}

1. Drew RAI, Romig M: Tropical Fruit Flies of South-East Asia (Teophritidae: Dacinae). Wallingford, Oxfordshire: CABl; 2013.

2. Drew RAl, Hancock DL: The Bactrocera dorsalis complex of fruit flies (Diptera: Tephritidae: Dacinae in Asia). Bull Entomol Res 1994, Supplement 2: 1-69.

3. Lux SA, Coperland RS, White IM, Manrakhan A, Billah MK: A new invasive fruit fly species from the Bactrocera dorsalis (Hendel) group detected in Africa. International Journal of Tropical Insect Science 2003, 23:355-361.

4. Drew RAI, Tsuruta K, White IM: A new species of pest fruit fly (Diptera : Tephritidae : Dacinae) from Sri Lanka and Africa. Afr Entomol 2005, 13:149-154.

5. Clarke AR, Armstrong KF, Carmichael AE, Milne JR, Raghu S, Roderick GK, Yeates DK: Invasive phytophagous pests arising through a recent tropical evolutionary radiation: The Bactrocera dorsalis complex of fruit flies. Annu Rev Entomol 2005, 50:293-319.

6. Iwahashi O: Aedeagal length of the oriental fruit fly, Bactrocera dorsalis (Hendel) (Diptera: Tephritidae), and its sympatric species in Thailand and the evolution of a longer and shorter aedeagus in the parapatric species of B. dorsalis. Appl Entomol Zool 2001, 36:289-297.

7. Khamis FM, Masiga DK, Mohamed SA, Salifu D, De Meyer M, Ekesi S: Taxonomic Identity of the Invasive Fruit Fly Pest, Bactrocera invadens: Concordance in Morphometry and DNA Barcoding. Plos One 2012, 7:e44862.

8. Krosch MN, Schutze MK, Armstrong KF, Boontop Y, Boykin LM, Chapman TA, Englezou A, Cameron SL, Clarke AR: Piecing together an integrative taxonomic puzzle: Microsatellite, wing shape and aedeagus length analyses of Bactrocera dorsalis s.l. (Diptera: Tephritidae) find no evidence of multiple lineages in a proposed contact zone along the Thai/Malay Peninsula. Syst Entomol 2012, 38:2-13.

9. Schutze MK, Krosch MN, Armstrong KF, Chapman TA, Englezou A, Chomic A, Cameron SL, Hailstones D, Clarke AR: Population structure of Bactrocera dorsalis s.s., B. papayae and B. philippinensis (Diptera: Tephritidae) in southeast Asia: Evidence for a single species hypothesis using mitochondrial DNA and wing-shape data. BMC Evol Biol 2012, 12:130.

10. Schutze MK, Jessup A, Clarke AR: Wing shape as a potential discriminator of morphologically similar pest taxa within the Bactrocera dorsalis species complex (Diptera: Tephritidae). Bull Entomol Res 2012, 102:103-111.

11. Schutze MK, Jessup A, Ul-Haq I, Vreysen MJB, Wornoayporn V, Vera MT, Clarke AR: Mating compatibility among four pest members of the Bactrocera dorsalis fruit fly species complex (Diptera: Tephritidae). J Econ Entomol 2013, 106:695-707.

12. Bo W, Ahmad S, Dammalage T, Tomas US, Wornoayporn V, UI Haq I, Cáceres C, Vreysen MJB, Hendrichs J, Schutze MK: Mating compatibility between Bactrocera invadens and Bactrocera dorsalis (Diptera: Tephritidae). J Econ Entomol 2014, 107:623-629.

13. Tan KH, Wee SL, Ono H, Nishida R: Comparison of methyl eugenol metabolites, mitochondrial COI, and rDNA sequences of Bactrocera philippinensis (Diptera: Tephritidae) with those of three other major pest species within the dorsalis complex. Appl Entomol Zool 2013, 48:275-282.
14. Wee SL, Hee AKW, Tan KH: Comparative sensitivity to and consumption of methyl eugenol in three Bactrocera dorsalis (Diptera: Tephritidae) complex sibling species. Chemoecology 2002, 12:193-197.

15. Tan KH, Tokushima I, Ono H, Nishida R: Comparison of phenylpropanoid volatiles in male rectal pheromone gland after methyl eugenol consumption, and molecular phylogenetic relationship of four global pest fruit fly species: Bactrocera invadens, B. dorsalis, B. correcta and $B$. zonata. Chemoecology 2011, 21:25-33.

16. Boykin LM, Schutze MK, Krosch MN, Chomic A, Chapman TA, Englezou A, Armstrong KF, Clarke AR, Hailstones D, Cameron SL: Multi-gene phylogenetic analysis of south-east Asian pest members of the Bactrocera dorsalis species complex (Diptera: Tephritidae) does not support current taxonomy. J Appl Entomol 2014, 138:235-253.

17. Frey JE, Guillen L, Frey B, Samietz J, Rull J, Aluja M: Developing diagnostic SNP panels for the identification of true fruit flies (Diptera: Tephritidae) within the limits of COI-based species delimitation. BMC Evol Biol 2013, 13:106.

18. Muraji M, Nakahara S: Phylogenetic relationships among fruit flies, Bactrocera (Diptera, Tephritidae), based on the mitochondrial rDNA sequences. Insect Mol Biol 2001, 10:549-559.

19. San Jose M, Leblanc L, Geib SM, Rubinoff D: An evaluation of the species status of Bactrocera invadens and the systematics of the Bactrocera dorsalis (Diptera: Tephritidae) complex. Ann Entomol Soc Am 2013, 106:684-694.

20. Aketarawong N, Isasawin S, Thanaphum S: Evidence of weak genetic structure and recent gene flow between Bactrocera dorsalis s.s. and $B$. papayae, across Southern Thailand and West Malaysia, supporting a single target pest for SIT applications. BMC Genet 2014, 15:70.

21. Franz G: Genetic sexing strains in Mediterranean fruit fly, an example for other species amenable to large-scale rearing as required for the sterile insect technique. In Sterile Insect Technique Principles and Practice in AreaWide Integrated Pest Management. The Netherlands: Springer;Dyck VA, Hendrichs J, Robinson AS. Dordrecht 2005:427-451.

22. Robinson AS: Development of genetic sexing strains for fruit fly sterile insect technology. Genetica 2002, 116:1-149.

23. Robinson AS: Genetic sexing strains in medfly, Ceratitis capitata, sterile insect technique programmes. Genetica 2002, 116:5-13.

24. Isasawin S, Aketarawong N, Thanaphum S: Characterization and evaluation of microsatellite markers in a strain of the oriental fruit fly, Bactrocera dorsalis (Diptera: Tephritidae), with a genetic sexing character used in sterile insect population control. Eur J Entomol 2012, 109:331-338.

25. Ji QE, Hou WR, Chen JH: Development of a genetic sexing strain and the sterile male technique of the Oriental fruit fly, Bactrocera dorsalis (Hendel). Acta Entomologica Sinica 2007, 50:1002-1008.

26. McCombs SD, Saul SH: Translocation-based genetic sexing system for the oriental fruit fly (Diptera: Tephritidae) based on pupal color dimorphism. Ann Entomol Soc Am 1995, 88:695-698.

27. Isasawin S, Aketarawong N, Lertsiri S, Thanaphum S: Development and characterization of genetic sexing strain in Bactrocera carambolae (Diptera: Tephritidae) by introgression for SIT. BMC Genet 2014, 15(Suppl 2):S2.

28. Mclnnis DO, Rendon P, Jang E, Sauers-Muller A, Sugayama R, Malavasi A Interspecific mating of introduced sterile Bactrocera dorsalis with wild $B$. carambolae (Diptera: Tephritidae) in Suriname: a potential case for crossspecies sterile insect technique. Ann Entomol Soc Am 1999, 92:758-765.

29. Noor MAF, Grams KL, Bertucci LA, Reiland J: Chromosomal inversions and the reproductive isolation of species. Proc Natl Acad Sci USA 2001, 98:12084-12088.

30. Ashburner M, Carson HL, Thompson J: The Genetics and Biology of Drosophila. London: Academic press INC; 1982.

31. Krimbas CB, Powell JR: Drosophila inversion polymorphism. USA: CRC Press, Inc.; 1992

32. Sturtevant $\mathrm{AH}$, Dobzhansky Th: Inversions in the third chromosome of wild race of Drosophila pseudoobscura, and their use in the study of the history of the species. Proc Natl Acad Sci USA 1936, 22:448.

33. Dobzhansky Th: The genetics and the origin of species. New York: Columbia University Press; 11936.

34. McGaugh SE, Noor MAF: Genomic impacts of chromosomal inversions in parapatric Drosophila species. Philos Trans R Soc London [ B-Biol] 2012, 367:422-429.

35. Stevison LS, Hoehn KB, Noor MAF: Effects of Inversions on Within- and Between-Species Recombination and Divergence. Genome Biology and Evolution 2011, 3:830-841 
36. Kulathinal RJ, Stevison LS, Noor MAF: The Genomics of Speciation in Drosophila: Diversity, Divergence, and Introgression Estimated Using Low-Coverage Genome Sequencing. Plos Genetics 2009, 5:e100550.

37. Noor MAF, Garfield DA, Schaeffer SW, Machado CA: Divergence between the Drosophila pseudoobscura and D. persimilis genome sequences in relation to chromosomal inversions. Genetics 2007, 177:1417-1428.

38. Noor MAF, Grams KL, Bertucci LA, Reiland J: Chromosomal inversions and the reproductive isolation of species. Proc Natl Acad Sci USA 2001, 98:12084-12088.

39. Ayala FJ, Coluzzi M: Chromosome speciation: Humans, Drosophila and mosquitoes. Proc Natl Acad Sci USA 2005, 102:6535-6542.

40. Manoukis NC, Powell JR, Toure MB, Sacko A, Edillo FE, Coulibaly MB, Traore SF, Taylor CE, Besansky NJ: A test of the chromosomal theory of ecotypic speciation in Anopheles gambiae. Proc Natl Acad Sci USA 2008, 105:2940-2945.

41. Lee Y, Collier TC, Sanford MR, Marsden CD, Fofana A, Cornel AJ, Lanzaro GC: Chromosome Inversions, Genomic Differentiation and Speciation in the African Malaria Mosquito Anopheles gambiae. Plos One 2013, 8:e57887.

42. Rieseberg LH: Chromosomal rearrangements and speciation. Trends Ecol Evolut 2001, 16:351-358.

43. Faria R, Navarro A: Chromosomal speciation revisited: rearranging theory with pieces of evidence. Trends Ecol Evolut 2010, 25:660-669.

44. Kirkpatrick M, Barton N: Chromosome inversions, local adaptation and speciation. Genetics 2006, 173:419-434.

45. Ranz JM, Maurin D, Chan YS, von Grotthuss M, Hillier LW, Roote J, Ashburner M, Bergman CM: Principles of genome evolution in the Drosophila melanogaster species group. PLoS Biol 2007, 5:1366-1381.

46. Carson HL: Inversions in Hawaiian Drosophila. In Drosophila inversion polymorphisms. Florida: CRC Press;'Krimbas CB, Powell JR. Boca Raton 1992:407-439.

47. Wasserman M: Cytological evolution of the Drosophila repleta species group. In Drosophila inversion polymorphism. Florida: CRC Press;Krimbas CB, Powell JR. Boca Raton 1992:455-552.

48. Zacharopoulou A: Polytene chromosome maps in the medfly Ceratitis capitata. Genome 1990, 33:184-197.

49. Mavragani-Tsipidou P, Karamanlidou G, Zacharopoulou A, Koliais S, Kastritsis C: Mitotic and polytene chromosome analysis in Dacus oleae (Diptera: Tephritidae). Genome 1992, 35:373-378.

50. Zacharopoulou A, Augustinos AA, Sayed WAA, Robinson AS, Franz G: Mitotic and polytene chromosomes analysis of the oriental fruit fly, Bactrocera dorsalis (Hendel) (Diptera: Tephritidae). Genetica 2011, 139:79-90.

51. Zacharopoulou A, Sayed WAA, Augustinos AA, Yesmin F, Robinson AS, Franz G: Analysis of mitotic and polytene chromosomes and photographic polytene chromosome maps in Bactrocera cucurbitae (Diptera: Tephritidae). Ann Entomol Soc Am 2011, 104:306-318.

52. Zambetaki A, Kleanthous K, Mavragani-Tsipidou P: Cytogenetic analysis of Malpighian tubule and salivery gland polytene chromosomes of Bactrocera oleae (Dacus oleae) (Diptera: Tephritidae). Genome 1995, 38:1070-1081.

53. Zhao JT, Frommer M, Sved JA, Zacharopoulou A: Mitotic and polytene chromosome analyses in the Queensland fruit fly, Bactrocera tryoni (Diptera: Tephritidae). Genome 1998, 41:510-526.

54. Drosopoulou E, Nestel D, Nakou I, Kounatidis I, Papadopoulos NT, Bourtzis K, Mavragani-Tsipidou P: Cytogenetic analysis of the Ethiopian fruit fly Dacus ciliatus (Diptera: Tephritidae). Genetica 2011, 139:723-732.

55. Drosopoulou E, Koeppler K, Kounatidis I, Nakou I, Papadopoulos NT, Bourtzis K, Mavragani-Tsipidou P: Genetic and cytogenetic analysis of the walnut-husk fly (Diptera: Tephritidae). Ann Entomol Soc Am 2010, 103:1003-1011.

56. Drosopoulou E, Augustinos AA, Nakou I, Koeppler K, Kounatidis I, Vogt H, Papadopoulos NT, Bourtzis K, Mavragani-Tsipidou P: Genetic and cytogenetic analysis of the American cherry fruit fly, Rhagoletis cingulata (Diptera: Tephritidae). Genetica 2011, 139:1449-1464.

57. Kounatidis I, Papadopoulos N, Bourtzis K, Mavragani-Tsipidou P: Genetic and cytogenetic analysis of the fruit fly Rhagoletis cerasi (Diptera: Tephritidae). Genome 2008, 51:479-491.

58. Garcia-Martinez V, Hernandez-Ortiz E, Zepeta-Cisneros CS, Robinson AS, Zacharopoulou A, Franz G: Mitotic and polytene chromosome analysis in the Mexican fruit fly, Anastrepha ludens (Loew) (Diptera: Tephritidae). Genome 2009, 52:20-30.
59. Zambetaki A, Zacharopoulou A, Scouras ZG, Mavragani-Tsipidou P: The genome of the olive fruit fly Bactrocera oleae: localization of molecular markers by in situ hybridisation to the salivary polytene chromosomes. Genome 1999, 42:744-751.

60. Drosopoulou E, Scouras ZG: The beta-tubulin gene familyeEvolution in the Drosophila montium subgroup of the melanogaster species group. Journal of Molecular Evolution 1995, 41:293-298.

61. Baimai V, Trinachartvanit W, Tigvattananont S, Grote PJ, Poramarcom R, Kijchalao U: Metaphase karyotypes of fruit flies of Thailand. I. Five sibling species of the Bactrocera dorsalis complex. Genome 1995, 38:1015-1022.

62. Zacharopoulou A, Franz G: Genetic and cytogenetic characterization of genetic sexing strains of Bactrocera dorsalis and Bactrocera cucurbitae (Diptera: Tephritidae). J Econ Entomol 2013, 106:995-1003.

63. Baimai V, Phinchongsakuldit J, Trinachartvanit W: Metaphase karyotypes of fruit flies of Thailand (III) Six members of the Bactrocera dorsalis complex. Zool Stud 1999, 38:110-118.

64. Baimai V, Sumrandee C, Tigvattananont S, Trinachartvanit W: Metaphase karyotypes of fruit flies of Thailand. V. Cytotaxonomy of ten additional new species of the Bactrocera dorsalis complex. Cytologia 2000, 65:409-417.

65. Baimai V: Heterochromatin accumulation and karyotypic evolution in some dipteran insects. Zoological Studies 1998, 37:75-88.

66. Yesmin F, Clyde MM: The chromosomes of the carambola fruit fly, Bactrocera carambolae (Diptera: Tephritidae): Metaphase karyotype and polytene genome. GSTF Journal of Bioscience 2012, 1:10-15.

67. Hernandez-Ortiz V, Bartolucci AF, Morales-Valles P, Frias D, Selivon D: Cryptic species of the Anastrepha fraterculus complex (Diptera: Tephritidae) : a multivarietie approach for the recognition of South American morphotypes. Ann Entomol Soc Am 2012, 105:305-318.

68. Bedo DG: Polytene chromosome mapping in Ceratitis capitata (Diptera: Tephritidae). Genome 1987, 29:598-611.

69. Caceres C, Segura DF, Vera MT, Wornoayporn V, Cladera JL, Teal P, Sapountzis P, Bourtzis K, Zacharopoulou A, Robinson AS: Incipient speciation revealed in Anastrepha fraterculus (Diptera; Tephritidae) by studies on mating compatibility, sex pheromones, hybridization, and cytology. Biol J Linn Soc 2009, 97:152-165.

70. Ohto K TEbina: Morphological characters and PCR-RFLP markers in the interspecific hybrids between Bactrocera carambolae and B. papayae of the $B$. dorsalis species complex (Diptera: Tephritidae). Research Bulletin of the Plant Protection Service of Japan 2006, 42:23-34.

71. Wee SL, Tan KH: Evidence of natural hybridization between two sympatric sibling species of Bactrocera dorsalis complex based on pheromone analysis. J Chem Ecol 2005, 31:845-858.

72. Research Project: SEQUENCING OF THE ORIENTAL FRUIT FLY (BACTROCERA DORSALIS) GENOME.[http://www.ars.usda.gov/research/ projects/projects.htm?accn_no $=420103]$.

73. Shen GM, Dou W, Niu JZ, Jiang HB, Yang WJ, Jia FX, Hu F, Cong L, Wang JJ: Transcriptome Analysis of the Oriental Fruit Fly (Bactrocera dorsalis). PLoS One 2011, 6:e29127.

74. Papadimitriou E, Kritikou D, Mavroidis M, Zacharopoulou A, Mintzas AC: The heat shock 70 gene family in the Mediterranean fruit fly Ceratitis capitata. Insect Mol Biol 1998, 7:279-290.

75. Zhao JT, Frommer M, Sved JA, Gillies CB: Genetic and molecular markers of the Queensland fruit fly, Bactrocera tryoni. J Hered 2003, 94:416-420.

76. Lagos D, Ruiz MF, Sanchez L, Komitopoulou K: Isolation and characterization of the Bactrocera oleae genes orthologous to the sex determining Sex-lethal and doublesex genes of Drosophila melanogaster. Gene 2005, 348:111-121.

77. Lagos D, Koukidou M, Savakis C, Komitopoulou K: The transformer gene in Bactrocera oleae: the genetic switch that determines its sex fate. Insect Mol Biol 2007, 16:221-230.

doi:10.1186/1471-2156-15-S2-S16

Cite this article as: Augustinos et al:: The Bactrocera dorsalis species complex: comparative cytogenetic analysis in support of Sterile Insect Technique applications. BMC Genetics 2014 15(Suppl 2):S16. 